\title{
Comprehensive study of phase transitions in relaxational systems with field-dependent coefficients
}

\author{
J. Buceta and Katja Lindenberg \\ Department of Chemistry and Biochemistry, and Institute for Nonlinear Science, \\ University of California San Diego, 9500 Gilman Drive, La Jolla, CA 92093-0340, USA
}

\begin{abstract}
We present a comprehensive study of phase transitions in single-field systems that relax to a nonequilibrium global steady state. The mechanism we focus on is not the so-called Stratonovich drift combined with collective effects, but is instead similar to the one associated with noise-induced transitions a la Horsthemke-Lefever in zero-dimensional systems. As a consequence, the noise interpretation (e.g., Itô vs Stratonvich) merely shifts the phase boundaries. With the help of a mean-field approximation, we present a broad qualitative picture of the various phase diagrams that can be found in these systems. To complement the theoretical analysis we present numerical simulations that confirm the findings of the mean-field theory.
\end{abstract}

PACS numbers: 05.40.-a, 05.10.Gg, 64.60.-i

\section{INTRODUCTION}

Equilibrium and non-equilibrium relaxational dynamics play an important role in many critical phenomena [1]. Typically, relaxational flows drive the evolution of the system to the equilibria determined by a Lyapunov energy functional $\mathcal{F}$ that depends on local potential functionals and on the interactions in the system [2, 3]. The celebrated $\phi^{4}$ functional is a paradigmatic example of such a potential functional and gives rise to well-known equilibrium models such as the so-called model A (a coarse-grained version of the Ising model), and model B (an archetype of phase separation dynamics), among others [1, 2].

Typical relaxational models describe the flow of a field $\varphi_{i}(t)$ defined on a $d$-dimensional square lattice via a Langevin equation of the form

$$
\dot{\varphi}_{i}(t)=-\Gamma \frac{\delta \mathcal{F}(\{\varphi\})}{\delta \varphi_{i}(t)}+\Gamma^{1 / 2} \xi_{i}(t)
$$

Here $i$ labels a lattice site, $\Gamma$ is a positive constant, $(\{\varphi\}) \equiv\left(\varphi_{1}, \ldots, \varphi_{N}\right)$ denotes the entire set of fields, and $\xi_{i}$ are Gaussian white noises with zero mean and correlation functions

$$
\left\langle\xi_{i}(t) \xi_{j}\left(t^{\prime}\right)\right\rangle=\sigma^{2} \delta_{i j} \delta\left(t-t^{\prime}\right) .
$$

Typically, the functional $\mathcal{F}$ consists of a local potential $V(\varphi)$ and an interaction term,

$$
\mathcal{F}(\{\varphi\})=\sum_{i}\left(V\left(\varphi_{i}\right)+\frac{K}{8 d} \sum_{\langle i j\rangle}\left(\varphi_{j}-\varphi_{i}\right)^{2}\right),
$$

where $K$ is the coupling coefficient. The left-most sum in Eq. (3) runs over all lattice sites and the right-most sum over the $2 d$ nearest neighbors of a given site $i$. A keystone in this formalism is the link (the "fluctuation-dissipation relation") between the intensity $\Gamma \sigma^{2}$ of the fluctuating contribution and the relaxation parameter $\Gamma$ through the constant $\sigma^{2}$ that in equilibrium systems is proportional to the temperature. The relaxation coefficient $\Gamma$ affects how fast the system relaxes to the global steady state.

Recent studies have revealed the importance of field-dependent relaxation coefficients $\Gamma(\varphi)$ in the dynamics of these systems [4]. A generic description for such systems is provided by the Langevin equation

$$
\dot{\varphi}_{i}(t)=-\Gamma\left(\varphi_{i}(t)\right) \frac{\delta \mathcal{F}(\{\varphi\})}{\delta \varphi_{i}(t)}+\left[\Gamma\left(\varphi_{i}(t)\right)\right]^{\frac{1}{2}} \xi_{i}(t),
$$

where $\Gamma(\varphi)$ and $[\Gamma(\varphi)]^{1 / 2}$ are both positive. For example, it has been shown that relaxational flows driven by fielddependent coefficients may present inverted phase diagrams where ordering effects increase with the intensity of the fluctuations. This behavior has been observed in polymer mixtures where spinodal decomposition, i.e., phase separation, increases with increasing temperature [5].

The importance of these flows may be even more pronounced in nonequilibrium systems, and goes well beyond the scenarios that lead to inverted phase diagrams. One example is that of pure noise-induced phase transitions [4]. 
Such phase transitions exhibit the striking feature that noise is the crucial element responsible for the appearance of ordered phases that disappear in the absence of noise. The first mechanism identified in the literature leading to such behavior [ 6 ] relied on a combination of the so-called Stratonovich drift that arises under this particular interpretation of the noise, and collective effects. The Stratonovich drift in these systems leads to opposite dynamical responses at short and long time scales. Collective effects generated by the coupling among the field elements can amplify short time instabilities (that would die away in the absence of coupling), thus leading to the observed noise-induced phase transitions. As a consequence, there was originally a widespread belief that noise-induced phase transitions could only be found in systems where there are no noise-induced transitions [7], since the latter transitions occur in zero-dimensional (uncoupled) systems. Recent studies involving relaxational flows with field-dependent relaxation coefficients have shown otherwise by presenting a system where both a transition (zero-dimensional) and a phasetransition (coupled systems) are induced by the same source of noise [4]. In fact, the mechanism is not attributable to the Stratonovich drift because these transitions occur independently of the noise interpretation [8]. Moreover, the same mechanism has been extended to pattern formation phenomena [9], generalizing a previous mechanism based on the Stratonovich drift [10].

A thorough understanding of relaxational models driven by field-dependent coefficients is therefore important for a number of reasons. They play a relevant role in critical phenomena, they may explain situations where inverted phase diagrams are obtained, and they constitute a generalization of the seminal work of Horsthemke and Lefever on noise-induced transitions to noise-induced phase transitions in coupled systems.

Whereas specific non-equilibrium relaxational models driven by field-dependent coefficients have been considered in the literature, herein we present a more general analysis of such models, whereby the specific cases considered earlier become part of a broad panorama. We ask two questions: (1) What are the circumstances (features of the model, values of the control parameters) that lead to purely noise-induced phase transitions? (2) What is the nature of the phases that can occur in these systems, and what are the features of the model that determine these phases? In answering these questions, we discuss the possible phase diagrams that can be obtained and show that their overall structure depends on geometrical properties such as the balance of convexities of the local potentials and of the fielddependent coefficients. Furthermore, we show how multistability can be induced by noise. Our point of departure for this analysis is the equation obtained by implementing the functional derivative of $\mathcal{F}$ indicated in Eq. (4),

$$
\dot{\varphi}_{i}(t)=\Gamma\left(\varphi_{i}\right)\left(-\frac{\partial V\left(\varphi_{i}\right)}{\partial \varphi_{i}}+\mathcal{L} \varphi_{i}\right)+\left[\Gamma\left(\varphi_{i}\right)\right]^{\frac{1}{2}} \xi_{i}(t),
$$

$\mathcal{L}$ being the discrete version of the diffusion Laplacian operator,

$$
\mathcal{L} \varphi_{i}=\frac{K}{2 d} \sum_{\langle i j\rangle}\left(\varphi_{j}-\varphi_{i}\right) .
$$

The paper is organized as follows. In Sec. II we construct the mean field approximation to Eq. (5) and establish the different phases that may appear in the model. A discussion of the possible phase boundaries between these phases, and the order of the transitions, are presented in Sec. III] The structure of the resulting phase diagrams is presented in Sec. IV as is a specific illustration that corroborates our more general analysis. Sections [II and IV]are supplemented by an appendix where we show that a particular type of transition, while it may occur, is necessarily an isolated point in the phase diagram. In Sec. $\nabla$ simulations of the full model corroborate some of our most striking results. We conclude in Sec. VI with a summary and some directions for future research.

\section{PHASE TRANSITIONS: MEAN-FIELD ANALYSIS}

We focus our analysis on systems that may undergo Ising-like phase transitions. A convenient order parameter to characterize the phase transitions is akin to the magnetization,

$$
m=|\langle\varphi\rangle|,
$$

where the brackets indicate both a spatial and a temporal average of the field in the steady state. Ordered states are associated with $m \neq 0$.

In order that a particular system described by Eq. (5) experience a phase transition driven by a spontaneous symmetry breaking of the order parameter from $m=0$ to $m \neq 0$, the symmetry that leads to $m=0$ must be embedded in the model. Note that if Eq. (5) is invariant under the combined transformation

$$
\begin{aligned}
\varphi & \leftrightarrow-\varphi, \\
\xi & \leftrightarrow-\xi
\end{aligned}
$$


then $m=0$, the symmetric state, is indeed always a solution for the order parameter. Equation (5) satisfies the required symmetry if $V(\varphi)$ and $\Gamma(\varphi)$ are even functions. We will thus consider this case throughout the paper. Furthermore, we also assume with no loss of generality that

$$
\begin{aligned}
& V(0)=0, \\
& \Gamma(0)=1 .
\end{aligned}
$$

The exact stationary probability density of Eq. (5) can be calculated for any noise interpretation, including the Itô and the Stratonovich interpretations [8]. However, any further analytic insights require further approximation. We implement a mean-field approximation in Eq. (5) by replacing the average value of the fields of the $2 d$ nearest-neighbors of any site $i$ by the mean field value $\langle\varphi\rangle$, that is,

$$
\frac{1}{2 d} \sum_{\langle i j\rangle} \varphi_{j} \rightarrow\langle\varphi\rangle .
$$

This procedure, which is equivalent to assuming global coupling rather than nearest neighbor coupling, disregards fluctuations of the neighboring sites around the mean value. Since all sites are then equivalent, the lattice index can be dropped and the set of field equations reduces to a single equation. However, the unknown mean value of the field appears in this equation and must be chosen self-consistently. Thus, we obtain a closed approximate version of the problem as expressed in the two equations

$$
\begin{aligned}
\dot{\varphi}(t) & =\Gamma(\varphi)\left(-\frac{\partial V(\varphi)}{\partial \varphi}+K(\langle\varphi\rangle-\varphi)\right)+[\Gamma(\varphi)]^{\frac{1}{2}} \xi(t), \\
\langle\varphi\rangle & =\langle\varphi\rangle_{\rho} .
\end{aligned}
$$

Here $\langle\cdot\rangle_{\rho}$ stands for a statistical average with respect to the stationary probability density associated with Eq. (11),

$$
\rho_{\mathrm{st}}(\varphi ;\langle\varphi\rangle)=N(\langle\varphi\rangle) \Gamma(\varphi)^{(\alpha-1)} e^{-\frac{2}{\sigma^{2}}\left(V(\varphi)+\frac{K}{2}(\langle\varphi\rangle-\varphi)^{2}\right)}
$$

$N(\langle\varphi\rangle)$ is the normalization constant, and $\alpha=0(\alpha=1 / 2)$ for the Itô (Stratonovich) interpretation of the noise. This mean field formulation can not be solved in full generality either, but it does allow some analytic characterization of the problem. It is this characterization that we pursue as far as possible.

Note that the disordered solution (symmetric state) $\langle\varphi\rangle=0$ always solves Eq. (12). Yet, other solutions such that $\langle\varphi\rangle \neq 0$ are also possible. We refer to the latter as ordered solutions. Note that as a consequence of the parity of the functions $V(\varphi)$ and $\Gamma(\varphi),\langle\varphi\rangle_{\rho}$ is an odd function of $\langle\varphi\rangle$ and therefore, if $\langle\varphi\rangle$ is a solution of Eq. (12), then so is $-\langle\varphi\rangle$. However, both lead to the same value of the order parameter $m$.

At this point, we must make a distinction between solutions and phases as determined by the stability of the former. We call a disordered $(D)$ phase a macroscopic state where $\langle\varphi\rangle=0$ is the only stable solution. If the solution $\langle\varphi\rangle=0$ is unstable, and only a solution with $\langle\varphi\rangle \neq 0$ is stable, the phase will be called ordered $(O)$. If $\langle\varphi\rangle=0$ coexists with other stable, but ordered, solutions, the phase will be denoted as multistable $(M)$.

Since the solution of the self-consistency equation (12) determines the acceptable values of $\langle\varphi\rangle$, it is crucial to understand the behavior of $\langle\varphi\rangle_{\rho}$ as a function of $\langle\varphi\rangle$. Noting that

$$
\frac{\partial\langle\varphi\rangle_{\rho}}{\partial\langle\varphi\rangle}=\frac{2 K}{\sigma^{2}}\left(\left\langle\varphi^{2}\right\rangle_{\rho}-\langle\varphi\rangle_{\rho}^{2}\right),
$$

and applying the generalized Schwarz inequality

$$
\left\langle f^{2}(\varphi)\right\rangle_{\rho}\left\langle g^{2}(\varphi)\right\rangle_{\rho} \geqslant\left|\langle f(\varphi) g(\varphi)\rangle_{\rho}\right|^{2}
$$

with $f(\varphi)=\varphi$ and $g(\varphi)=1$, one concludes that the right hand side of Eq. (14) is positive, and therefore $\langle\varphi\rangle_{\rho}$ is a monotonically increasing function of $\langle\varphi\rangle$. Moreover, taking the limit of

$$
\left\langle\varphi^{n}\right\rangle_{\rho}=\int_{-\infty}^{\infty} d \varphi \varphi^{n} \rho_{\mathrm{st}}(\varphi ;\langle\varphi\rangle)
$$

as $\langle\varphi\rangle \rightarrow \pm \infty$ immediately leads to

$$
\lim _{\langle\varphi\rangle \rightarrow \pm \infty}\left\langle\varphi^{n}\right\rangle_{\rho} \rightarrow\langle\varphi\rangle_{\rho}^{n},
$$


and, consequently,

$$
\lim _{\langle\varphi\rangle \rightarrow \pm \infty} \frac{\partial\langle\varphi\rangle_{\rho}}{\partial\langle\varphi\rangle}=0
$$

Therefore it follows that

$$
\lim _{\langle\varphi\rangle \rightarrow \infty}\langle\varphi\rangle_{\rho}<\langle\varphi\rangle, \quad \lim _{\langle\varphi\rangle \rightarrow-\infty}\langle\varphi\rangle_{\rho}>\langle\varphi\rangle
$$

that is, $\langle\varphi\rangle_{\rho}$ necessarily lies below (above) $\langle\varphi\rangle$ as $\langle\varphi\rangle$ goes to plus (minus) infinity.

Figure 1 illustrates the resulting possible different phases in terms of the possible solutions of the self-consistency equation (12). The dashed lines represent $\langle\varphi\rangle$, the solid curves $\langle\varphi\rangle_{\rho}$, and the self-consistency solutions are their points of intersection. Our subsequent discussions presume that the system definitely goes to a stable state. We exclude "runaway" systems that do not fall into this category.

\section{PHASE BOUNDARIES: SECOND AND FIRST ORDER PHASE TRANSITIONS}

With Fig. 1in mind, consider now the possible resulting behaviors of the order parameter as we transition from one phase to another by changing a control parameter. Note that the general model (5) (as well as the mean field version of the model) depends on only two parameters, the coupling coefficient $K$ and the noise intensity $\sigma^{2}$, so the control parameter that characterizes a change from one phase to another could be either of these two (or some combination of them). The question then is how the points of intersection in Fig. 1 move as one varies a control parameter that takes the system from the behavior shown in one panel to that shown in another.

Consider the transition as a system moves from the behavior shown in panel (a) of Fig. 1 to that of panel (b). The way this is expected to occur is that the solid curve rotates upward so that in addition to the $\langle\varphi\rangle=0$ solution, another solution emerges at the origin. This second solution then moves upward along the diagonal as the control parameter increases. This $(\mathrm{a}) \rightarrow(\mathrm{b})$ transition is illustrated in the top panel of Fig. 2 where the solid lines represent stable solutions and the dotted lines the unstable solutions. The transition between the disordered and ordered phases is continuous in the order parameter (second order phase transition). A transition from panel (b) in Fig. 11 to panel (c) would involve the evolution of a kink in the curve that first cuts the diagonal at the origin and then moves upward toward the existing cut. Associated with this there is a change in the curvature of $\langle\varphi\rangle_{\rho}$ near the origin. This transition is sketched in the middle panel of Fig. 2] where we show the two nonzero solutions moving closer together as the control parameter increases. The transition between the ordered and multistable phases is discontinuous (first order phase transition) and is expected to exhibit hysteresis. The discontinuity is clearly apparent, for example, in the jump from the disordered branch of the multistable phase as one decreases the control parameter and this branch becomes unstable. Finally, a transition from panel (a) to panel (c), the bottom panel of Fig. 2 again involves a change in the curvature near the origin and the evolution of a kink that first touches the diagonal at a single nonzero value that then separates into two as the control parameter increases. The transition between disordered and multistable phases is again discontinuous. Note that all of these transitions can also proceed in the opposite direction than illustrated here, e.g. a transition from order to disorder would occur as in the top panel but from right to left.

We stress that the transitions between disordered and ordered phases are continuous in the order parameter (second order phase transitions), whereas the transitions from or to multistable phases are discontinuous (first order) and are therefore expected to exhibit hysteresis. There may be singular isolated exceptions to this latter conclusion, as discussed in the Appendix.

A more quantitative characterization of the phase transitions is possible for those transitions that involve a change in the stability properties of the $\langle\varphi\rangle_{\rho}=0$ solution, that is, for phase transitions between disordered and ordered phases, and between ordered and multistable phases (i.e., the top and middle panels in Fig. 2). This characterization involves the first two nonzero derivatives of $\langle\varphi\rangle_{\rho}$ with respect to $\langle\varphi\rangle$ in the vicinity of the origin. The first derivative provides information about the slope of $\langle\varphi\rangle_{\rho}$ and the third about the concavity/convexity $\left(\langle\varphi\rangle_{\rho}\right.$ is an odd function of $\langle\varphi\rangle$, so even derivatives around the origin vanish). Transitions between disordered and ordered phases (second order) occur when

$$
\begin{gathered}
\left.\frac{\partial\langle\varphi\rangle_{\rho}}{\partial\langle\varphi\rangle}\right|_{\langle\varphi\rangle=0}=1 \\
\left.\frac{\partial^{3}\langle\varphi\rangle_{\rho}}{\partial\langle\varphi\rangle^{3}}\right|_{\langle\varphi\rangle=0}<0
\end{gathered}
$$




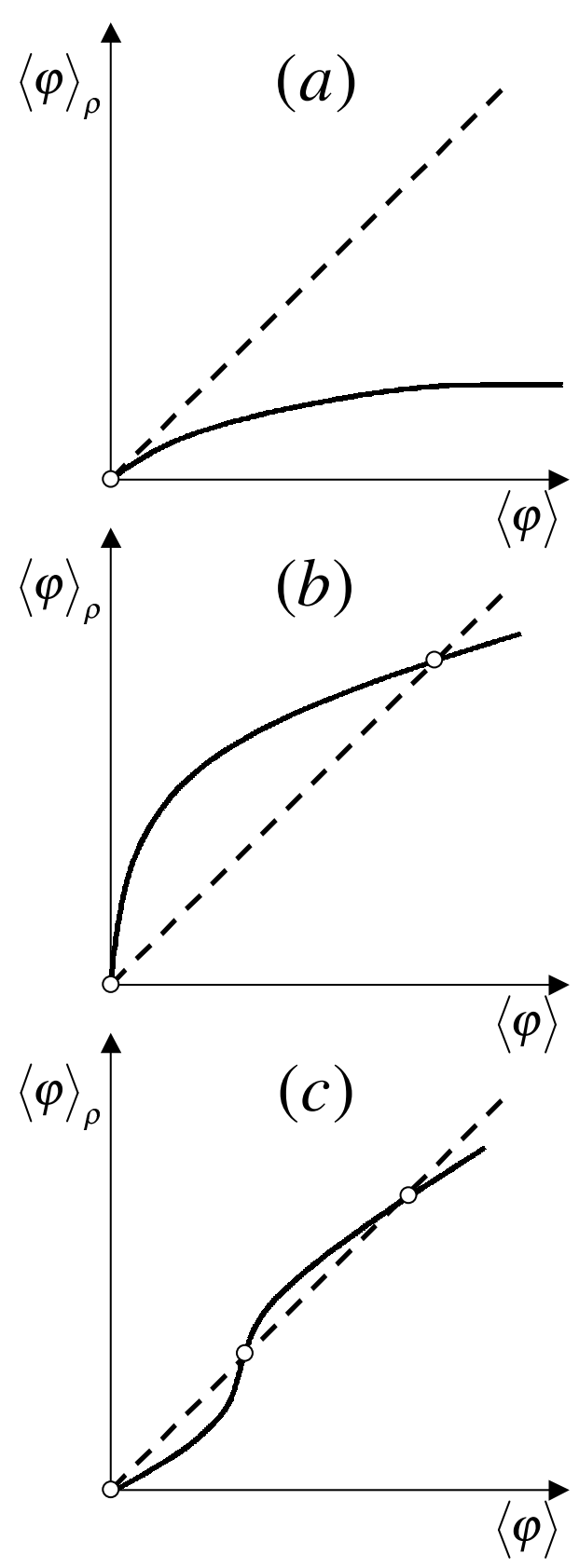

FIG. 1: Schematic of the possible solutions for the self-consistency equation (12). If the only solution is $\langle\varphi\rangle=0$, panel (a), the system is in a disordered phase. In panel (b), there is another solution in addition to the one at $\langle\varphi\rangle=0$. Only the nonzero solution is stable, so that this represents an ordered phase. In panel (c) there is an even number of nonzero solutions (here, two) in addition to the $\langle\varphi\rangle=0$ solution. The $\langle\varphi\rangle=0$ solution is stable, the next intersection is unstable, and the third is again a stable solution. The system thus exhibits multistability in this case, and involves the coexistence of a disordered and an ordered solution.

Transitions between ordered and multistable phases (first order) occur when

$$
\begin{gathered}
\left.\frac{\partial\langle\varphi\rangle_{\rho}}{\partial\langle\varphi\rangle}\right|_{\langle\varphi\rangle=0}=1, \\
\left.\frac{\partial^{3}\langle\varphi\rangle_{\rho}}{\partial\langle\varphi\rangle^{3}}\right|_{\langle\varphi\rangle=0}>0 .
\end{gathered}
$$

In turn, these derivatives can be expressed in terms of the cumulants of the probability density $\rho_{\text {st }}(\varphi ; 0)$. We proceed 

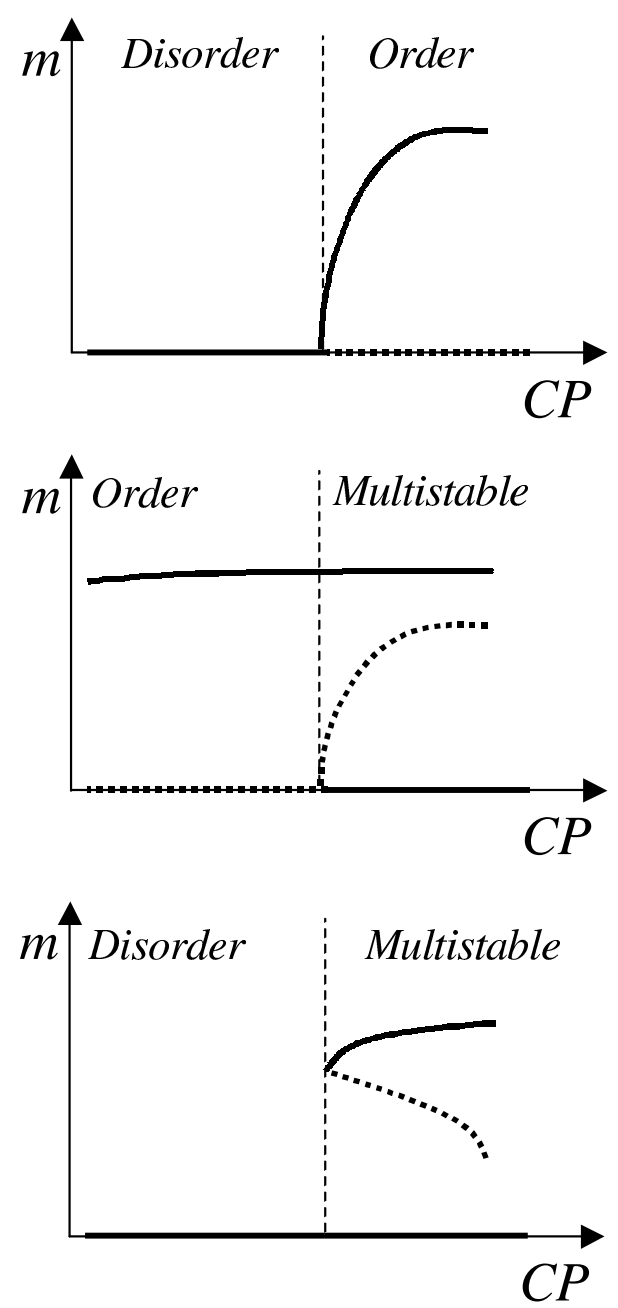

FIG. 2: Behavior of the order parameter, $m$, as a function of a control parameter, $C P$, in the vicinity of a transition point. Transitions between disordered and ordered phases are always second order (top panel). As shown in the middle and bottom panels, phase transitions from, or to, multistable phases are (with the exception of singular points discussed in the Appendix) first order.

to establish this relation.

Since $\langle\varphi\rangle_{\rho}$ is an odd function of $\langle\varphi\rangle$, its Taylor expansion around the solution $\langle\varphi\rangle=0$ reads

$$
\langle\varphi\rangle_{\rho}=\sum_{n=0}^{\infty} a_{2 n+1}\langle\varphi\rangle^{2 n+1}
$$

where

$$
a_{2 n+1}=\left.\frac{1}{(2 n+1) !} \int_{-\infty}^{\infty} \frac{\partial^{2 n+1} \rho_{\mathrm{st}}(\varphi ;\langle\varphi\rangle)}{\partial\langle\varphi\rangle^{2 n+1}}\right|_{\langle\varphi\rangle=0} \varphi d \varphi
$$

Furthermore, a straightforward but tedious calculation leads to the result

$$
a_{2 n+1}=\frac{2^{2 n+1}}{(2 n+1) !}\left(\frac{K}{\sigma^{2}}\right)^{2 n+1} C_{2 n+2},
$$

where $C_{2 n+2}$ is the $(2 n+2)^{\mathrm{th}}$ cumulant of the probability distribution $\rho_{\mathrm{st}}(\varphi ; 0)$. The relation of the cumulants to the 
statistical moments of the probability distribution is given by

$$
C_{2 n+2}=-\left|\begin{array}{ccccccc}
0 & 1 & 0 & 0 & 0 & 0 & \cdots \\
\left\langle\varphi^{2}\right\rangle_{0} & 0 & 1 & 0 & 0 & 0 & \ddots \\
0 & \left\langle\varphi^{2}\right\rangle_{0} & 0 & 1 & 0 & 0 & \ddots \\
\left\langle\varphi^{4}\right\rangle_{0} & 0 & \left(\begin{array}{l}
3 \\
1
\end{array}\right)\left\langle\varphi^{2}\right\rangle_{0} & 0 & 1 & 0 & \ddots \\
0 & \left\langle\varphi^{4}\right\rangle_{0} & 0 & \left(\begin{array}{l}
4 \\
2
\end{array}\right)\left\langle\varphi^{2}\right\rangle_{0} & 0 & 1 & \ddots \\
\left\langle\varphi^{6}\right\rangle_{0} & 0 & \left(\begin{array}{c}
5 \\
1
\end{array}\right)\left\langle\varphi^{4}\right\rangle_{0} & 0 & \left(\begin{array}{c}
5 \\
3
\end{array}\right)\left\langle\varphi^{2}\right\rangle_{0} & 0 & \ddots \\
\vdots & \ddots & \ddots & \ddots & \ddots & \ddots & \ddots
\end{array}\right|_{2 n+2}
$$

where $|\cdot|_{2 n+2}$ indicates the determinant of the $(2 n+2) \times(2 n+2)$ matrix, $(\cdot)$ the binomial coefficients, and $\langle\cdot\rangle_{0}$ stands for statistical averages over the probability density $\rho_{\mathrm{st}}(\varphi ; 0)$. Therefore, around $\langle\varphi\rangle=0$ the self-consistency equation reads,

$$
\langle\varphi\rangle=\langle\varphi\rangle_{\rho}=\langle\varphi\rangle \sum_{n=0}^{\infty} \frac{2^{2 n+1}}{(2 n+1) !}\left(\frac{K}{\sigma^{2}}\right)^{2 n+1} C_{2 n+2}\langle\varphi\rangle^{2 n}
$$

and it then follows that

$$
\begin{aligned}
\left.\frac{\partial\langle\varphi\rangle_{\rho}}{\partial\langle\varphi\rangle}\right|_{\langle\varphi\rangle=0} & =\frac{2 K}{\sigma^{2}} C_{2}, \\
\left.\frac{\partial^{3}\langle\varphi\rangle_{\rho}}{\partial\langle\varphi\rangle^{3}}\right|_{\langle\varphi\rangle=0} & =8\left(\frac{K}{\sigma^{2}}\right)^{3} C_{4} .
\end{aligned}
$$

In summary, the boundaries between disordered and ordered phases and between ordered and multistable phases are characterized by the first two nonzero cumulants of the probability distribution $\rho_{\text {st }}(\varphi ; 0)$ as follows:

$$
\begin{gathered}
\left.\begin{array}{c}
C_{2}=\frac{\sigma^{2}}{2 K} \\
C_{4}<0
\end{array}\right\} \Longrightarrow \text { Second order phase transition (order-disorder boundary) } \\
\left.\begin{array}{c}
C_{2}=\frac{\sigma^{2}}{2 K} \\
C_{4}>0
\end{array}\right\} \Longrightarrow \text { First order phase transition (order-multistable boundary). }
\end{gathered}
$$

Transitions between disordered and multistable phases can not be characterized in this fashion since they require information about $\langle\varphi\rangle_{\rho}$ away from $\langle\varphi\rangle=0$.

\section{OVERALL STRUCTURE OF PHASE DIAGRAMS}

Having discussed the possible phase transitions that might be observed in the mean field system (11)-(12), we now ask which particular phases might be present for particular values of the control parameters $K$ and $\sigma^{2}$. In this section we present an analytic deduction of the phases present for small and for large values of the coupling coefficient $K$. The behavior for intermediate values must be deduced on the basis of plausibility arguments that we introduce later.

We start by defining

$$
\Theta_{K}=a_{1}-1=\frac{2 K}{\sigma^{2}} C_{2}-1
$$

This quantity measures the differences in the slopes of $\langle\varphi\rangle$ and $\langle\varphi\rangle_{\rho}$ as a function of $\langle\varphi\rangle$ near the origin. According to the analysis presented in the previous section, $\Theta_{K}>0$ for an ordered phase and $\Theta_{K}<0$ in either disordered or multistable regions of the phase diagram. Moreover, the phase boundaries to or from ordered states are given by the zeros of $\Theta_{K}$.

Note that $\Theta_{0}=-1$, that is, $\langle\varphi\rangle_{\rho}=0$ in the absence of coupling. Thus, $\langle\varphi\rangle=0$ is the only possible solution to the self-consistency equation (12) in the small coupling limit, and the system is disordered in this limit. At sufficiently weak coupling the system is therefore always disordered. 
One can easily check that

$$
\left.\frac{\partial \Theta_{K}}{\partial K}\right|_{K=0}>0
$$

Thus, as $K$ grows from zero the system advances toward the ordered phase. This statement does not mean that the system will actually enter into the ordered phase as the coupling increases; it simply states the ordering role of weak but increasing coupling.

On the other hand and more interestingly, it is possible to investigate the strong coupling limit as follows. We first introduce the convenient notation

$$
\begin{aligned}
\Phi(\varphi) & =\Gamma(\varphi)^{\alpha-1} e^{-\frac{2}{\sigma^{2}} V(\varphi)} \\
I_{2 n}\left(K, \sigma^{2}\right) & =\int_{-\infty}^{\infty} \varphi^{2 n} \Phi(\varphi) e^{-\frac{K}{\sigma^{2}} \varphi^{2}} d \varphi
\end{aligned}
$$

where $n \geqslant 0$. We can then write all the non-zero moments of $\rho_{\text {st }}(\varphi ; 0)$ as $\left\langle\varphi^{2 n}\right\rangle_{0}=I_{2 n} / I_{0}$. In particular, the cumulants of interest here can be written as

$$
C_{2}=\frac{I_{2}}{I_{0}}, \quad C_{4}=\frac{I_{4}}{I_{0}}-3 \frac{I_{2}^{2}}{I_{0}^{2}} .
$$

Moreover, notice that all the moments can be reduced to the calculation of $I_{0}$ since

$$
I_{2 n}\left(K, \sigma^{2}\right)=(-1)^{n} \sigma^{2 n} \frac{\partial^{n} I_{0}\left(K, \sigma^{2}\right)}{\partial K^{n}} .
$$

A series expansion of $I_{0}$ useful for large values of $K$ follows from an expansion of $\Phi(\varphi)$ around $\varphi=0$, which allows us to carry out the integral:

$$
I_{0}\left(K, \sigma^{2}\right)=\int_{-\infty}^{\infty} \sum_{m=0}^{\infty}\left(\frac{\Phi^{(m)}}{m !} \varphi^{m}\right) e^{-\frac{K}{\sigma^{2}} \varphi^{2}} d \varphi=\sum_{m=0}^{\infty} \frac{\Phi^{(2 m)} \pi^{1 / 2}}{m ! 2^{2 m}}\left(\frac{\sigma^{2}}{K}\right)^{m+1 / 2}
$$

where,

$$
\Phi^{(z)}=\left.\frac{\partial^{z} \Phi(\varphi)}{\partial \phi^{z}}\right|_{\varphi=0}
$$

Introducing Eq. (37) into Eq. (36), we obtain the series

$$
I_{2 n}\left(K, \sigma^{2}\right)=\sum_{m=0}^{\infty} \frac{\Phi^{(2 m)} \pi^{1 / 2}[m+1 / 2]_{n}}{m ! 2^{2 m}}\left(\frac{\sigma^{2}}{K}\right)^{m+n+1 / 2}
$$

where

$$
[z]_{n}=\prod_{l=0}^{n-1}(z+l), \quad[z]_{0}=1
$$

The moments of $\rho_{\text {st }}(\varphi ; 0)$ then read

$$
\left\langle\varphi^{2 n}\right\rangle_{0}=\left(\frac{\sigma^{2}}{K}\right)^{n} \frac{\sum_{m=0}^{\infty} \frac{\Phi^{(2 m)}}{m ! 2^{2 m}}[m+1 / 2]_{n}\left(\frac{\sigma^{2}}{K}\right)^{m}}{\sum_{m=0}^{\infty} \frac{\Phi^{(2 m)}}{m ! 2^{2 m}}\left(\frac{\sigma^{2}}{K}\right)^{m}} .
$$


A more convenient expression for Eq. (41) is obtained by performing its Taylor expansion around $\left(\sigma^{2} / K\right) \rightarrow 0$,

$$
\begin{aligned}
\left\langle\varphi^{2 n}\right\rangle_{0} \underset{K \gg 1}{\overline{=}} & \left(\frac{\sigma^{2}}{K}\right)^{n}\left[[1 / 2]_{n}+\left(\frac{\sigma^{2}}{K}\right) \frac{\Phi^{(2)}}{8 \Phi^{(0)}}\left([3 / 2]_{n}-[1 / 2]_{n}\right)\right. \\
& +\left(\frac{\sigma^{2}}{K}\right)^{2} \frac{1}{32\left(\Phi^{(0)}\right)^{2}}\left(\Phi^{(4)} \Phi^{(0)}\left([5 / 2]_{n}-[1 / 2]_{n}\right)\right. \\
& \left.\left.-2\left(\Phi^{(2)}\right)^{2}\left([3 / 2]_{n}-[1 / 2]_{n}\right)\right)+\mathcal{O}\left(\left(\frac{\sigma^{2}}{K}\right)^{3}\right)\right] .
\end{aligned}
$$

The first term in the series (42), i.e., up to order $\left(\sigma^{2} / K\right)^{n}$, leads to the familiar result of applying the steepest descent method [12] to $I_{0}$,

$$
\left\langle\varphi^{2 n}\right\rangle_{0} \underset{K \rightarrow \infty}{=}\left(\frac{\sigma^{2}}{K}\right)^{n}[1 / 2]_{n}
$$

However, this result is not sufficiently accurate to capture enough of the large-coupling behavior of $\left\langle\varphi^{2 n}\right\rangle_{0}$ and shed light on the behavior of the phase boundaries in that limit. Keeping up to the next order, that is,

$$
\left\langle\varphi^{2 n}\right\rangle_{0} \underset{K \gg 1}{=}\left(\frac{\sigma^{2}}{K}\right)^{n}\left[[1 / 2]_{n}+\left(\frac{\sigma^{2}}{K}\right) \frac{\Phi^{(2)}}{8 \Phi^{(0)}}\left([3 / 2]_{n}-[1 / 2]_{n}\right)+\mathcal{O}\left(\left(\frac{\sigma^{2}}{K}\right)^{2}\right)\right],
$$

one finds for the function $\Theta_{K}$

$$
\Theta_{K \gg 1}=\frac{1}{2}\left(\frac{\sigma^{2}}{K}\right) \frac{\Phi^{(2)}}{\Phi^{(0)}}+\mathcal{O}\left(\left(\frac{\sigma^{2}}{K}\right)^{2}\right) .
$$

Note that $\Phi^{(0)}=1$ [c.f. Eq. (9)], and therefore the sign of $\Theta_{K}$ for large values of the coupling is determined by the sign of $\Phi^{(2)}$,

$$
\Phi^{(2)}=(\alpha-1) \Gamma^{(2)}-\frac{2}{\sigma^{2}} V^{(2)}
$$

If $\Phi^{(2)}>0$ then $\Theta_{K \gg 1}>0$ (ordered phase). On the other hand, if $\Phi^{(2)}<0$ then $\Theta_{K \gg 1}<0$ (disordered or multistable phase). That is, whether or not the system is in an ordered phase depends only on the balance of convexities of the local potential and the field-dependent coefficient at the origin.

Furthermore, since $\Theta_{K \gg 1}=\mathcal{O}\left(\left(\sigma^{2} / K\right)\right)$, which vanishes as $K$ increases, one knows that for large coupling the system is "near" a phase boundary of an ordered phase. One can gain some insight into the type of transition that might be involved by studying the fourth cumulant, $C_{4}$ [cf. Eqs.(29) and (301)]. Using Eq. (42) in Eq. (35) and recalling that $\Phi^{(0)}=1$, we obtain,

$$
C_{4}(K \gg 1)=\frac{1}{16}\left(\frac{\sigma^{2}}{K}\right)^{4}\left(\Phi^{(4)}-3\left(\Phi^{(2)}\right)^{2}\right)+\mathcal{O}\left(\left(\frac{\sigma^{2}}{K}\right)^{5}\right)
$$

where,

$$
\begin{aligned}
\Phi^{(4)}= & -\frac{12}{\sigma^{2}}(\alpha-1) V^{(2)} \Gamma^{(2)}+\frac{12}{\sigma^{4}}\left(V^{(2)}\right)^{2}+(\alpha-1) \Gamma^{(4)} \\
& +3(\alpha-1)(\alpha-2)\left(\Gamma^{(2)}\right)^{2}-\frac{2}{\sigma^{2}} V^{(4)} .
\end{aligned}
$$

We thus confirm that, independently of the behavior of the system at intermediate values of the coupling, for large coupling the appearance or disappearance of ordered phases as reflected in the sign of the fourth cumulant depends on the geometrical properties of $V(\varphi)$ and of $\Gamma(\varphi)$ around the origin.

There are basically only two distinct generic types of behavior of these functions around the origin, and therefore only four possible combinations. The possible types of functions are shown in Fig. [3 where we have plotted the simple 


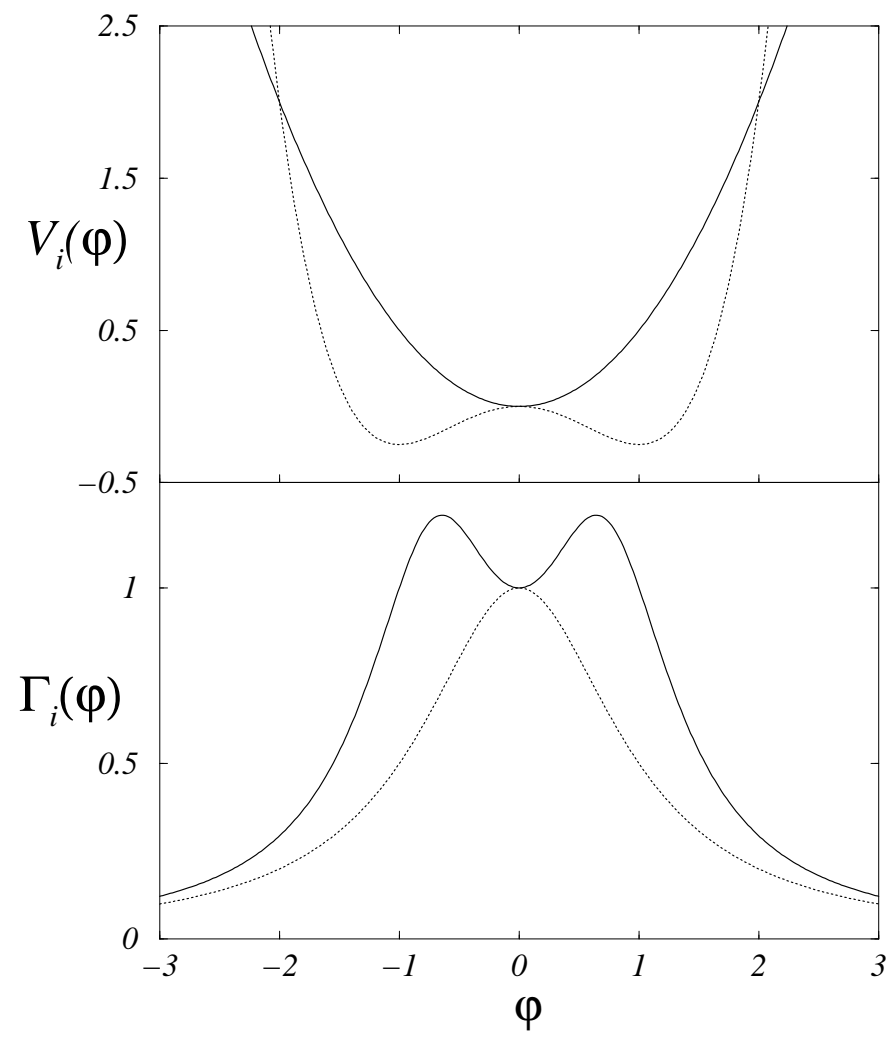

FIG. 3: Generic local potentials $V_{i}(\varphi)$ and field-dependent coefficients $\Gamma_{i}(\varphi)$ as a function of the field $\varphi$. The solid lines are for $i=1$ and the dotted lines for $i=2$. The behavior at the origin of the derivatives of $V(\varphi)$ and $\Gamma(\varphi)$ determine the phase at large values of the coupling (see text).

representative cases 13

$$
\begin{aligned}
& V_{1}(\varphi)=\frac{\varphi^{2}}{2} \\
& V_{2}(\varphi)=\frac{\varphi^{4}}{4}-\frac{\varphi^{2}}{2}
\end{aligned}
$$

and

$$
\begin{aligned}
\Gamma_{1}(\varphi) & =\frac{1+\varphi^{2}}{1+\varphi^{4}} \\
\Gamma_{2}(\varphi) & =\frac{1}{1+\varphi^{2}} .
\end{aligned}
$$

Note that the field-dependent coefficient $\Gamma_{1}$ favors fluctuations around $\varphi= \pm 1$ while $\Gamma_{2}$ leads to the largest fluctuations around $\varphi=0$ [4]. It is quite straightforward to determine the sign of $C_{4}$ on the basis of the derivatives of these functions (one can use the generic forms (49) and (50) as a guide) and compile the following table. We emphasize that these are results in the strong coupling limit. The entries in the table indicate order $(O)$, disorder $(D)$, and multistability $(M)$ :

\begin{tabular}{|c|c|c|}
\hline & $V_{1}(\varphi)$ & $V_{2}(\varphi)$ \\
\hline$\Gamma_{1}(\varphi)$ & $M$ & $\begin{array}{c}O \text { if } \sigma^{2}<\sigma_{c}^{2} \\
M \text { if } \sigma^{2}>\sigma_{c}^{2}\end{array}$ \\
\hline$\Gamma_{2}(\varphi)$ & $\begin{array}{r}O \text { if } \sigma^{2}>\sigma_{c}^{2} \\
D \text { if } \sigma^{2}<\sigma_{c}^{2}\end{array}$ & $O$ \\
\hline
\end{tabular}

Here $\sigma_{c}^{2}$ is a critical value of the noise intensity that separates different phases; for the generic models displayed above, $\sigma_{c}^{2}=1 /(1-\alpha)$. Note that the noise interpretation through the value of $\alpha$ simply shifts the critical value of $\sigma_{c}^{2}$. The 
case $V_{1}, \Gamma_{2}$ with the functions given above has recently been studied in the context of phase transitions and pattern formation 4, 8, 9]. Also, in agreement with our general analysis it was noted recently [8] that in that particular case the noise-induced phase transition is not attributable to the so-called Stratonovich drift, as is the case in other noise-induced phenomena [6].

We have thus arrived at the generic phase structure for the mean field problem (11)-(12) in the weak coupling limit (the system is disordered) and in the strong coupling limit (as shown in the table). For intermediate coupling we are not able to provide a general quantitative analysis except to note that if $\sigma^{2} \rightarrow 0$, the system is always in a disordered $(D)$ phase since the fluctuations are needed to provide the energy to induce symmetry breakings.

Despite this difficulty, one can introduce compelling arguments to connect the phase behavior that we have established in these limits, and to arrive at a set of full phase diagrams.

There are several unknown regions connecting various known phases at this point. In the $V_{1}, \Gamma_{1}$ case we need to connect the weak-coupling $D$ phase to the strong-coupling $M$ phase. For the $V_{2}, \Gamma_{1}$ combination we require a connection between the $D$ phase and an $O$ phase if the noise is weak, or to an $M$ phase if it is strong. With the $V_{1}, \Gamma_{2}$ combination the connection needs to be made between the disordered weak-coupling phase and an ordered or a disordered strong-coupling phase depending on the noise intensity. And in the $V_{2}, \Gamma_{2}$ case a connection needs to be established from the disordered to the ordered phase. The simplest possible scenarios for connections are the following. The simplest connection between disordered phases is simply a disordered phase, i.e., a situation where no phase transition occurs at all. A connection between disordered and ordered phases is most straightforwardly accomplished through a single second-order phase transition. Finally, for the connection between disordered and multistable phases, two different scenarios are most feasible. One possibility is that the connection is mediated through an ordered phase, as follows. As noted in Fig. 2] when a multistable region appears from a disordered phase, the unstable solution tends at first to move downward as the control parameter increases. If the unstable solution eventually vanishes, the disordered phase necessarily becomes unstable and one necessarily enters an ordered phase. Such destabilization does not occur if the transition is mediated by and ordered phase. On the contrary, multistable phases arising from $O M$ transitions grow more stable as the control parameter increases (see Fig. 2). In this case, a feasible sequence would be of the form $D O M$. On the other hand, a direct $D M$ transition may also occur, but only if the initial vanishing tendency of the unstable solution is stabilized as the coupling increases.

We can corroborate this scenario by calculating the phase diagrams that are obtained from the mean field approximation for the particular functions (49) and (50). We numerically solve the self-consistency equation (12) and compute the boundaries separating different phases. Recall that the noise interpretation simply shifts the transitions points but does not change the phase diagram structure. We present the results for $\alpha=0$, that is, the Itô interpretation, for which $\sigma_{c}^{2}=1$. The results are shown in Fig. 4.

Note that the actual structure of the phase diagrams at small and large values of the couplings is perfectly captured by our analysis. Moreover, the value of the critical noise intensity is $\sigma_{c}^{2}=1$, as predicted. As for the unknown domains shown for intermediate coupling, our arguments about the simplest scenarios agree with the mean-field results. For example, for the $V_{2}, \Gamma_{1}$ combination the appearance of the multistable phase with increasing $K$ is mediated through an ordered phase. We point out that for the case $V_{1}, \Gamma_{1}$, there is a triple point where all phases merge. At this critical point a continuous phase transition between disordered and multistable phases occurs. As noted earlier, this behavior is singular and isolated. Note also that multistability appears in this case by means of a DOM sequence with increasing coupling $K$ for noise intensities to the right of the triple point. However, to the left of the triple point the multistable phase arises from a $D M$ sequence. Moreover, above the triple point and with increasing $\sigma^{2}$, multistable phases are always destabilized and followed by an ordered phase, as mentioned above. There is evidently an asymptote at $\sigma^{2} \simeq 2$ for the phase boundary separating the disordered and multistable phases. However, this critical noise intensity is not captured by our theory since it does not involve slopes and convexities near the origin. Instead, we show below how this second critical value of the noise intensity can be calculated by analyzing the zero-dimensional version of the problem.

A number of other striking features of the phase diagrams are noteworthy. For both $V_{1}$ cases, independently of the value of the coupling, the system becomes more ordered as the noise intensity increases. In the case of $\Gamma_{1}$ this behavior is associated with the destabilization of multistable phases and in general suggests that the phenomenon of the so-called inverted phase diagrams depends mainly on the convexity of the local potential around the origin. It is also worth noting the phenomenon of reentrant noise-induced multistability for the case $V_{2}, \Gamma_{1}$. If the coupling is greater than $K \sim 10$, increasing the noise intensity causes a transition from an ordered phase to a multistable phase. However a further increase in the noise intensity eventually leads the system back to the ordered phase. This behavior resembles the phenomenon of reentrance as a function of the intensity of the fluctuations in other noise-induced phenomena [6, 10]. However, in the latter the phase changes are from disordered to ordered and, for sufficiently intense noise, back to disorder.

Zero-Dimensional Analysis. Previous studies for the particular case $V_{1}, \Gamma_{2}$ [4, 8] have revealed that in the case of relaxational-flows with field dependent relaxation coefficients the mechanism responsible for the phase transition is 


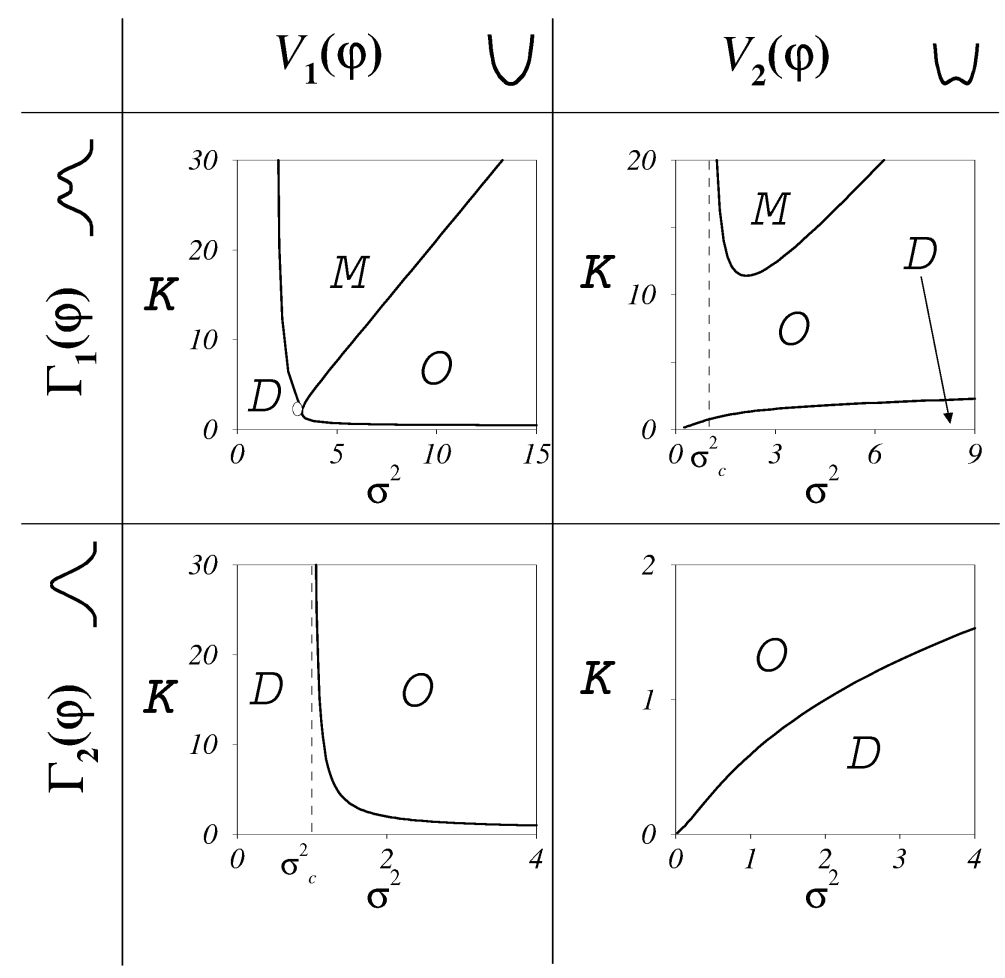

FIG. 4: Mean-field phase diagrams as a function of the local potentials and field-dependent kinetic coefficients given by Eqs. (49) and (50). The small open circle in the phase diagram for $\Gamma_{1}$ and $V_{1}$ where the three phases merge indicates an isolated singular critical point (triple point) where a continuous phase transition between disordered and ordered phases occurs (see the appendix). The overall structure of the phase diagrams is in perfect agreement with the schematic structures discussed in the text.

similar to that which drives the noise-induced transition a la Horsthemke-Lefever in zero-dimensional systems [7]. In the zero-dimensional case, noise-induced transitions are associated with changes in the extrema of the local potential. We present an analysis of the zero-dimensional system to compare with some of the results of our mean-field analysis. The zero-dimensional version of Eq. (5) reads

$$
\dot{\varphi}(t)=\Gamma(\varphi)\left(-\frac{\partial V(\varphi)}{\partial \varphi}\right)+[\Gamma(\varphi)]^{\frac{1}{2}} \xi(t)
$$

that is, the uncoupled version of our original model (5). The stationary probability density now is

$$
\rho_{\mathrm{st}}(\varphi)=N e^{-\frac{2}{\sigma^{2}} V_{\mathrm{eff}}(\varphi)},
$$

where $N$ is the normalization constant and $V_{\text {eff }}(\varphi)$ is the effective potential,

$$
V_{\mathrm{eff}}(\varphi)=V(\varphi)+\frac{\sigma^{2}(1-\alpha)}{2} \ln \Gamma(\varphi)
$$

The equilibria of the effective potential are given by the condition $V_{\mathrm{eff}}^{\prime}\left(\varphi^{*}\right)=0$, that is,

$$
\Gamma\left(\varphi^{*}\right) V^{\prime}\left(\varphi^{*}\right)+\frac{\sigma^{2}(1-\alpha)}{2} \Gamma^{\prime}\left(\varphi^{*}\right)=0 .
$$

The stability of the equilibria depends on the sign of the second derivative of the potential at the equilibrium points. A noise-induced transition occurs when there is a change in the stability of the solution $\varphi^{*}$. Therefore, the boundary of stability is given by $V_{\text {eff }}^{\prime \prime}\left(\varphi^{*}\right)=0$, that is,

$$
\Gamma^{2}\left(\varphi^{*}\right) V^{\prime \prime}\left(\varphi^{*}\right)+\frac{\sigma^{2}(1-\alpha)}{2}\left(\Gamma\left(\varphi^{*}\right) \Gamma^{\prime \prime}\left(\varphi^{*}\right)-\left(\Gamma^{\prime}\left(\varphi^{*}\right)\right)^{2}\right)=0 .
$$


Note that $\varphi^{*}=0$ is always a solution of Eq. (54) and therefore the disordered solution is stable if

$$
V^{\prime \prime}(0)+\frac{\sigma^{2}(1-\alpha)}{2} \Gamma^{\prime \prime}(0)=0 .
$$

This equation corresponds exactly to the stability boundary associated with Eq. (46) . In other words, the strongly coupled system behaves exactly as the uncoupled system. In the cases $V_{2}(\varphi), \Gamma_{1}(\varphi)$ and $V_{1}(\varphi), \Gamma_{2}(\varphi)$ the critical value of the noise intensity that changes the stability of the disordered solution to an ordered one is exactly as calculated in the coupled system, $\sigma_{c}^{2}=1 /(1-\alpha)$.

Moreover, our purpose in analyzing the zero-dimensional system is also to understand the phase boundary that separates multistable and disordered phases in the case $V_{1}, \Gamma_{1}$ in the spatially extended problem. Recall that in that case the stability of the disordered solution does not change, and therefore we are not able to use our mean field analysis near the disordered state to compute phase boundaries. However, we can use Eqs. (54) and (55) to support our numerical findings that indicated that there is a critical noise intensity separating those two phases. While solving Eq. (54) for solutions $\varphi^{*} \neq 0$ is rather cumbersome and Eq. (55) does not have an analytic solution for those values, it is trivial to solve the problem numerically. The result agrees perfectly with our previous findings: there is an asymptote at $\sigma^{2} \simeq 2$ (Itô) that corresponds to the critical value of the noise intensity separating disordered and multistable phases.

\section{NUMERICAL SIMULATIONS}

To check the predictions of the mean field theory we present numerical simulations of Eq. (51) in a two dimensional square lattice with nearest neighbor interactions, the Itô interpretation of the noise, and periodic boundary conditions. We focus on the case $V_{1}, \Gamma_{1}$. This is the most interesting, previously unexplored, case: a striking effect of the noise, a noise-induced multistable phase and an inverted phase diagram, occur in this case. Note that the case $V_{1}, \Gamma_{2}$ has been studied recently [4], the case $V_{2}, \Gamma_{2}$ presents a phase diagram with a phenomenology similar to the well-known model A [1, 14], and the case $V_{2}, \Gamma_{1}$ presents as its main feature the same striking phenomenology of noise-induced multistability as does the case $V_{1}, \Gamma_{1}$.

Figure 5 shows the order parameter $m$ as a function of the noise intensity $\sigma^{2}$ for a fixed value of the coupling constant, $K=10$. The system is seen to explore the three possible phases, disordered, multistable, and ordered, as the fluctuations become stronger. Moreover, the system presents an inverted phase diagram where order becomes more prominent as the noise is increased. As indicated by the discontinuous behavior of the order parameter, the phase transitions are first order in all cases. To detect the multistable phase and the associated hysteresis, we integrated Eq. (5) under two different conditions. The initial conditions and the noise realizations are identical in both cases. A difficulty in such simulations is that it takes an inordinately long time to reach a steady state (eventually the system leaves any steady state if the system is finite, but this time can be made as long as desired by increasing the size of the system). To overcome this difficulty, in one case we added a very small external field that favors the solution $m=0$ while in the other we added one that favors an ordered solution. As soon as steady states were reached, the external fields were turned-off. The insets of Fig. [5 show, by means of a density plot, the values of the field in the multistable phase for the points $A$ and $B$ for which $\sigma^{2} \approx 3$. The scale of the density plots is also presented and is the same for both insets. The insets highlight the striking feature of the noise-induced multistability and show the two possible states within the multistable region.

\section{CONCLUSIONS}

We have presented a detailed studied of phase transitions in models with field-dependent relaxation coefficients. By means of a mean-field approximation in combination with other analytical techniques and plausibility arguments supported by particular examples, we have elucidated the phase diagrams that can be found in such models. We stress that our methods can easily be applied to a variety of other systems. Moreover, we have demonstrated that disorder-multistability continuous phase transitions are singular points in the phase diagram, and that the phases for large values of the coupling are determined by geometrical features of the local potential and of the field-dependent coefficient in the vicinity of the origin. We have also showed that the behavior of the extended system at large values of the coupling coefficient is equivalent to the behavior of the uncoupled zero-dimensional system. Therefore, the mechanism responsible for the phase transitions is similar to the noise-induced transitions a la Horsthemke-Lefever, and is not attributable to the Stratonovich drift together with collective effects involved in other noise-induced phenomena 6, 10]. Finally, we have performed numerical simulations of a particular case to check the results of the mean-field approximation. The numerical results are in qualitative agreement with the theoretical predictions and 


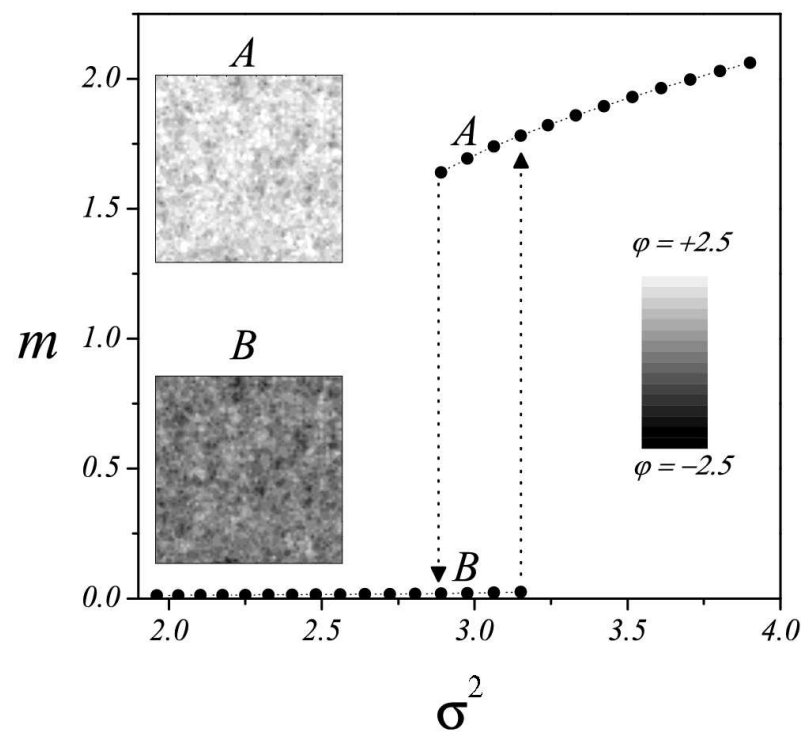

FIG. 5: Order parameter, $m$, as a function of the noise intensity, $\sigma^{2}$, for the case $V_{1}, \Gamma_{1}$ and a fixed value of the coupling $K=10$. The noise term is interpreted in the Itô sense. Note that the system explores the three possible phases (disordered, multistable, and ordered) as the intensity of the noise is increased. The insets show the steady states of the field for the points $A$ and $B$ indicated in the figure (see text). The noise intensity at these points is $\sigma^{2} \approx 3$.

reproduce the main features of the system, most notably, the occurrence of noise-induced multistability, and of an inverted phase diagram indicating that stronger noise induces greater order.

We envision further modifications of these models that would extend the richness of the observed phenomenology. In particular, including other degrees of freedom and considering different couplings increases the complexity of the multistability phenomena caused by the noise. Further degrees of freedom could, for example, lead to locking of the system either in an oscillatory mode (limit-cycle) or in a stationary state (focus) depending on the initial conditions. Considering couplings that favor morphological instabilities could lead to pattern formation determined entirely by the initial conditions. Work in these directions is in progress.

\section{Acknowledgments}

This work was partially supported by the Engineering Research Program of the Office of Basic Energy Sciences at the U. S. Department of Energy under Grant No. DE-FG03-86ER13606, by MECD-Spain Grant No. EX2001-02880680, and by MCYT-Spain Grant No. BFM2001-0291.

\section{APPENDIX A: SINGULAR TRANSITION}

In Fig. 2 and the discussion surrounding it we noted that with the exception of singular points in the parameter space, phase transitions from disorder to multistability are first order (discontinuous). In this appendix we expand on this assertion. By "singular" we mean that if there is a point $\left(\sigma_{*}^{2}, K_{*}\right)$ in the phase diagram where a continuous transition between disorder and multistability exists, then no neighboring points in the phase diagram can present the same transition. In other words, continuous disorder-multistability phase transitions are isolated critical points in the phase diagram, and there is no plausible continuous function $K\left(\sigma^{2}\right)$ connecting them.

Figure [ s shows the behavior of the order parameter $m$ as a function of a control parameter in the vicinity of a continuous disorder-multistability phase transition. Note that for that behavior to happen, all three roots of the selfconsistency equation must vanish exactly at the critical value of the control parameter. Moreover, the convexity of $\langle\varphi\rangle_{\rho}$ must also change sign at exactly that value. If such a critical point $\left(\sigma_{*}^{2}, K_{*}\right)$ exists, it must satisfy the conditions 


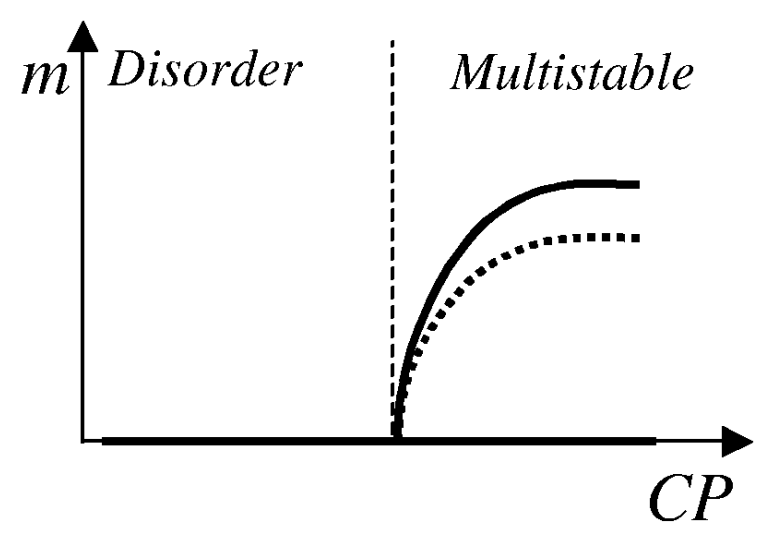

FIG. 6: Schematic behavior of the order parameter as a function of a control parameter in the vicinity of a continuous disorder-multistability phase transition. This kind of behavior is singular and isolated in the phase diagram.

$$
\left.\begin{array}{c}
C_{2}=\frac{1}{2}\left(\frac{\sigma_{*}^{2}}{K_{*}}\right) \\
C_{4}=0
\end{array}\right\} \Longrightarrow\left\{\begin{array}{c}
\left\langle\varphi^{2}\right\rangle_{0}=\frac{1}{2}\left(\frac{\sigma_{*}^{2}}{K_{*}}\right) \\
\left\langle\varphi^{4}\right\rangle_{0}=\frac{3}{4}\left(\frac{\sigma_{*}^{2}}{K_{*}}\right)^{2}
\end{array} .\right.
$$

These requirements fulfill the Schwarz inequality, Eq. (15), and we must therefore conclude that such a critical point is possible.

Now, assume the existence of the critical point $\left(\sigma_{*}^{2}, K_{*}\right)$. We investigate the requirements for a neighboring point $\left(\sigma_{*}^{2}+\varepsilon_{\sigma^{2}}, K_{*}+\varepsilon_{K}\right)$ to also be associated with a disorder-multistability phase transition. A straightforward calculation leads to the following condition to be satisfied by $\varepsilon_{\sigma^{2}}$,

$$
\varepsilon_{\sigma^{2}}\left(\left\langle\varphi^{2}\right\rangle_{0}\langle V(\varphi)\rangle_{0}-\left\langle\varphi^{2} V(\varphi)\right\rangle_{0}\right)=0
$$

This can in general only be satisfied if $\varepsilon_{\sigma^{2}}=0$. As for $\varepsilon_{K}$, it must satisfy

$$
\varepsilon_{K}\left(\left\langle\varphi^{4}\right\rangle_{0}\left\langle\varphi^{2}\right\rangle_{0}-\left\langle\varphi^{6}\right\rangle_{0}\right)+\varepsilon_{K} \frac{3}{2}\left(\frac{\sigma^{2}}{K}\right)^{3}=0
$$

Again, the only acceptable solution to this equation is $\varepsilon_{K}=0$. Therefore, if there exists a critical point in the phase diagram where a continuous disorder-multistability phase transition occurs, that point is singular in the sense that it is isolated.

[1] P.C. Hohenberg, and B.I. Halperin, Rev. Mod. Phys. 49, 435 (1977).

[2] J. García-Ojalvo and J.M. Sancho, Noise in Spatially Extended Systems (Springer, New York, 1999).

[3] R. Montagne, E. Hernández-García, and M. San Miguel, Physica D 96, 47 (1996).

[4] M. Ibañes, J. García-Ojalvo, R. Toral, and J.M. Sancho, Phys. Rev. Lett. 87, 020601 (2001).

[5] H.L. Snyder, P. Meakin, and S. Reich, Macromolecules 16, 757 (1983).

[6] C. Van den Broeck, J.M.R. Parrondo, and R. Toral, Phys. Rev. Lett. 73, 3395 (1994); C. Van den Broeck, J.M.R. Parrondo, R. Toral, and R. Kawai, Phys. Rev. E 55, 4084 (1997).

[7] W. Horsthemke and R. Lefever, Noise-Induced Transitions (Springer, Berlin, 1984).

[8] O. Carrillo, M. Ibañes, J. García-Ojalvo, J. Casademunt, and J.M Sancho, Phys. Rev. E 67, 046110 (2003).

[9] J. Buceta, M. Ibañes, J.M. Sancho, and K. Lindenberg, Phys. Rev. E 67, 021113 (2003).

[10] J.M.R. Parrondo, C. Van den Broeck, J. Buceta, and F.J. de la Rubia, Physica A 224, 153 (1996).

[11] K. Kitahara and M. Imada, Supp. Prog. Theo. Phys. 64, 65 (1978).

[12] G. Arfken, Mathematical Methods for Physicists (Academic Press, Orlando, 1985).

[13] Note that the present analysis is also applicable to equilibrium models with $\Gamma=1$, which leads to to the so-called Gaussian model when $V=V_{1}$ and to model A if the local potential is $V_{2}$.

[14] R. Toral and A. Chakrabarti, Phys. Rev. B 42, 2445 (1990). 